\title{
Less sickness with more motion and/or mental distraction
}

\author{
Jelte E. Bos ${ }^{a, b}$ \\ ${ }^{\mathrm{a}}$ TNO Perceptual and Cognitive Systems, P.O. Box 23, 3769 ZG Soesterberg, Netherlands \\ ${ }^{\mathrm{b}}$ Research Institute MOVE, Faculty of Human Movement Sciences, VU University, Amsterdam, Netherlands \\ Tel.: +31888665925;E-mail: Jelte.Bos@tno.nl
}

Received 23 January 2014

Accepted 16 June 2014

\begin{abstract}
.
BACKGROUND: Motion sickness may reduce passenger comfort and crew performance. Countermeasures are dominated by medication with specific and often undesirable side effects.

OBJECTIVE: To shown that sickness due to motion can be reduced by adding an inherent non-sickening vibration and by mental distraction.

METHODS: Eighteen blindfolded subjects were exposed to 20 minutes of off-vertical axis rotation (OVAR). Vibration was added by means of a head rest. Effects of OVAR and vibration were tested separately and in combination, while the subjects were performing an audio letter memorising task. This task was absent to test the effect of mental distraction. Effects were quantified by rating subjective misery and objective task performance.

RESULTS: Sixteen subjects completed the experiment and showed in mutual comparable conditions that head vibration reduced the amount of sickness by $25 \%$, the mental distraction did so by $19 \%$, and the combined effect resulted in a reduction of $39 \%$. Their task performance decreased with increasing sickness.

CONCLUSION: Sickness due to low-frequency motion can be reduced by adding a high-frequency vibration and by mental distraction. The effect of vibration can be understood by assuming an internal model used by the central nervous system to optimise the control of body motion.
\end{abstract}

Keywords: Motion sickness, nausea, vibration, performance, mental distraction

\section{Introduction}

With varying susceptibility, most people suffer from motion sickness in some way [54 pp. 38-54]. This not only concerns physical motion in different environments [17/23]30|42-44|62]63], but also visual motion [20[39]. People without organs of balance have been shown to be insensitive to both physical [35|49] as well as visual motion [15], although some sickness in labyrinthine defective patients cannot be ruled out [36]. While motion sickness is mostly considered a comfort issue, it also affects task performance [4]10, 49], thus not only being a luxury factor, but an economic factor as well.
Although medication has been proven to be effective in a large number of conditions [46]60] it is not always the desired countermeasure due to a number of side effects such as a dry mouth and drowsiness, the latter also affecting task performance. Apart from synthetic drugs, ginger, not having the mentioned side effects, seems to be effective too [28[45]. Although cognitive and biofeedback methods, including that using controlled breathing and listening to (pleasant) music have also been shown to be effective against motion sickness, these methods require a form of training and/or attention during the motion exposure [18]37|38|40|61|64|65]. Other incidental publications report on the beneficial effects of viewing an artificial horizon [57], viewing a more complex artificial 
3D Earth-fixed frame of reference, possibly including an anticipatory trajectory [22]33], applying stroboscopic vision [55], or lowering the environmental temperature [47]. The effect of acupressure or electrical acustimulation still remains doubtful [2|14]. In conclusion there does not seem to be one countermeasure eliminating all symptoms of motion sickness, but a combination of remedies may be satisfactory.

The current paper therefore presents a yet unreported remedy by applying high-frequency vibration to the head, the idea being threefold. By personal experience it appeared that while suffering from carsickness, some comfort can be obtained by leaning the head against a vibrating car or bus window. Another idea uses the argument that motion sickness only occurs at motion frequencies typically below $1 \mathrm{~Hz}$, while higher frequency vibration is not sickening [26/43|44|50]. Adding non-sickening high-frequency vibration to a sickening low - (i.e., low-frequency) frequency motion may then decrease the overall sensitivity of the system, thus resulting in less sickness in effect. A more advanced argument, lastly, is based on the hypothesis that motion sickness is the result of a conflict between a sense of verticality as determined by integrated sensory information and an expectation thereof as determined by an internal or observer model [3|5-7| $9|5|$ | In this theory, the conflict is dominated by a phase lag due to a process making a distinction between gravitational and inertial accelerations as sensed by the otoliths, and this conflict can be attenuated by decreasing an internal feedback or Kalman [59] gain. The addition of non-sickening random noise to the input of the sensory signals may thus decrease the amount of sickness caused by low-frequency motion. Irrespective its explanation, the main hypothesis studied in this paper is that the sickening effect of low-frequency motion, in this case off-vertical axis rotation (OVAR), can be reduced by adding high-frequency vibration. This paper, consequently, does not examine how vibration is transferred from seat to head or vice versa, and the consequences thereof for application. Apart from a second issue discussed below, it only examines whether or not high-frequency vibration is capable of reducing sickness induced by low-frequency motion.

The second assumption considered, concerns the effect of distraction by mental activity on sickness severity [1]3158], the most extensive review still given in [54, pp. 26, 71-73, 240]. The latter assumed that "motion sickness is less likely to occur when attention is directed toward external events than when the subjects are asked to turn the 'mental eye' inwards and re-
Table 1

Experimental conditions

\begin{tabular}{cccc}
\hline Condition & OVAR & Head vibration & Task \\
\hline A & - & + & + \\
B & + & - & + \\
C & + & + & + \\
D & + & - & - \\
\hline
\end{tabular}

port upon their own feelings". Data from the literature, however, are ambiguous. In [29], for example, a positive effect of a visually guided problem solving task was found as opposed to performing no task with the eyes closed, whereas in [61] no differences were found between the mean number of rotations achieved in a Coriolis stress test performed with and without a cognitive task. Within both studies, however, stimulus conditions varied. By contrast, [16] observed that sickness symptoms tended to increase when asking subjects to concentrate upon the imposed motion. To reduce a possible variability in motion sickness severity due to a variability in mental state, and to address the issue per se, a demanding mental task with the additional advantage of yielding a number of performance measures, was therefore considered in the current study as well.

\section{Methods}

\subsection{Experimental design}

Three main factors were considered in this study: OVAR, head vibration and a task performed. Eight trials would consequently be required to realise a complete factorial design. To prevent confounding effects by sickness accumulation and habituation [54], each condition should be realised on a separate day. In this case that would be eight days per subject. Though not impossible, that would yet be a rather impracticable design. The experiment was therefore limited to four conditions with and/or without the sub-factors OVAR, head vibration, and a task, as listed Table 1 . These conditions were then applied in a fixed order, however, all conditions having been applied first, second, third, and last equally often as described further below. To avoid possible interference and thus confounding by visual cues, subjects completed all conditions blindfolded.

\subsection{Off-vertical axis rotation}

Off-vertical axis rotation (OVAR) was used in this experiment to make people sick. The advantage of using OVAR is that subjects cannot withdraw from it 

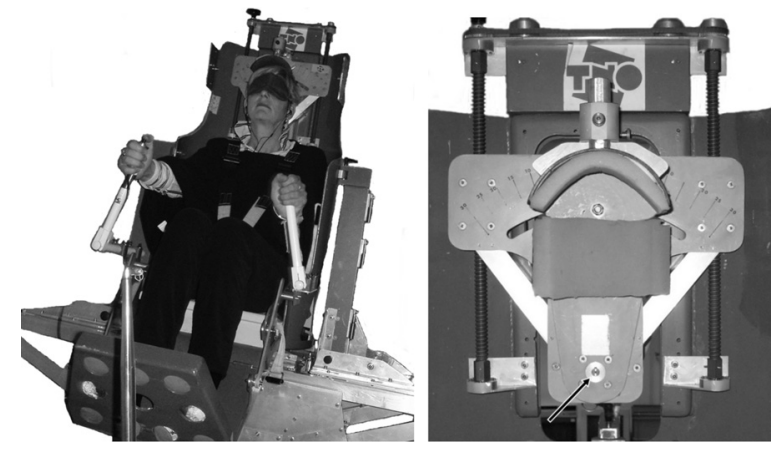

Fig. 1. Modified Barany chair with head, foot, and hand rests, and a subject wearing an eye mask (left). Laterally movable headrest with rotation axis indicated by the arrow (right).

willingly or even reduce the effects thereof. The latter may happen by reducing head movements during cross-coupled or Coriolis manoeuvres, for example. Another advantage of using OVAR is its high level of controllability by means of only two parameters: the angle of the rotation axis with respect to Earth and the rotation velocity. In this way, sickness severity and the accumulation of sickness over time can be chosen experimentally. Based on work by [17], $10^{\circ}$ of tilt and $60 \%$ angular velocity were assumed to elicit moderate levels of sickness within a period of 20 minutes. A moderate level was preferred because subjects were to be exposed to the sickening stimulus three times (i.e., in conditions $\mathrm{B}, \mathrm{C}$, and $\mathrm{D}$ ) within a few days and not to withdraw due to excessive misery. An exposure time of 20 minutes was chosen to include the onset of sickness as thought typical for carsickness.

A modified Barany chair was used for inducing this OVAR stimulus as shown in Fig. 1. Subjects wore a five-point safety belt and kept their head on axis by means of an adjustable headrest (see below). Chair rotation was started and stopped using an acceleration and deceleration of $3 \% \mathrm{~s}^{2}$, also in cases subjects asked for a premature ending due to sickness. When no OVAR was applied (condition A), the rotation chair was deliberately positioned upright. Only then does the average acceleration due to gravity in the transverse plane equal zero, as it does during OVAR, a static tilt resulting in a static asymmetric acceleration in that plane, rather different from that experienced during OVAR [32].

\subsection{Head vibration}

The Barany chair was equipped with a servo controlled movable head rest, allowing head roll (cervi-
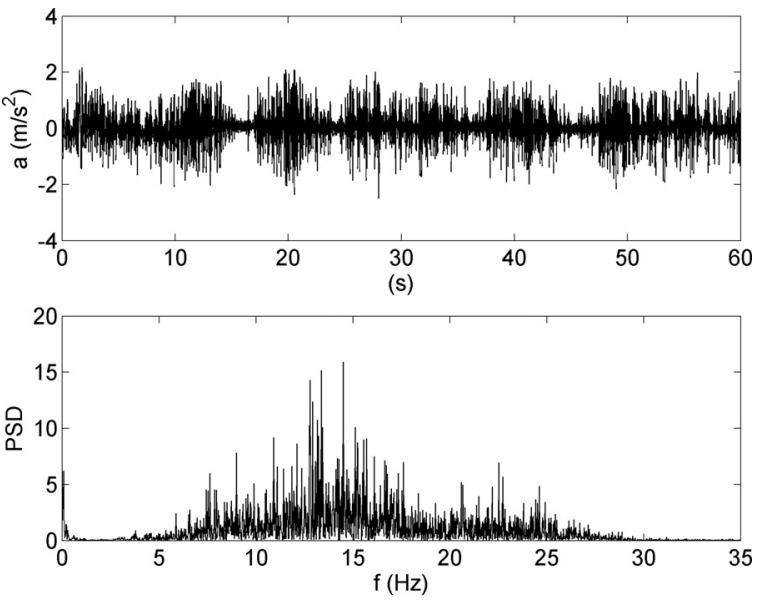

Fig. 2. Individual record of lateral head vibration acceleration (top) and the accompanying power spectral density (PSD, bottom).

cal lateral flexion) to the left and right about an axis fixed to $24 \mathrm{~cm}$ below the apex of the head (see Fig. 1). Two head restraints were used, consisting of circular curved foam padded plates, $7 \mathrm{~cm}$ wide and extending $14 \mathrm{~cm}$, one on top and one at the back of the head. To mimic the vibration one may experience when leaning the head against a car or coach window, white noise was band pass filtered between $10 \mathrm{~Hz}$ and $30 \mathrm{~Hz}$. The amplitude of this signal was subsequently quasi randomly modulated with a sum of sinuses with arbitrarily chosen frequencies of $1 / 6 \mathrm{~Hz}, 1 / 7 \mathrm{~Hz}$ and $1 / 10 \mathrm{~Hz}$ to enhance the "road feeling" of the signal. Actual head tilt was limited to $1^{\circ}$. Using an accelerometer clenched between the teeth of two test subjects, an estimate of actual head vibration was obtained, showing lateral linear RMS accelerations of $0.37 \mathrm{~m} / \mathrm{s}^{2}$ and $0.57 \mathrm{~m} / \mathrm{s}^{2}$. These levels are about half the maximum allowable value and about equal to the action value as mentioned in [21] when lasting for eight hours. They are about a quarter of the caution level mentioned in [34], which is applicable to the lumbar spine and the connected nervous system when it would last for 30 minutes. A typical time trace and the corresponding power spectral density are shown in Fig. 2. By adding this (relatively) high-frequency motion to the low-frequency OVAR motion, the two frequency contents being mutually exclusive, "more" motion here relates to the overall acceleration amplitude, the frequencies included, and the degrees of motion freedom involved.

\subsection{Sickness}

Subjective misery was recorded by means of an 11point misery scale (MISC, Table 2, [12[13]). The ad- 
Table 2

11-point MIsery SCale (MISC)

\begin{tabular}{llc}
\hline Symptoms & & MISC \\
\hline No problems & & 0 \\
Some discomfort, but no specific symptoms & & 1 \\
Dizziness, cold/warm, headache, & Vague & 2 \\
stomach/throat awareness, sweating, & Little & 3 \\
blurred vision, yawning, burping, & Rather & 4 \\
tiredness, salivation, ... but no nausea & Severe & 5 \\
& Little & 6 \\
Nausea & Rather & 7 \\
& Severe & 8 \\
& Retching & 9 \\
Vomiting & & 10 \\
\hline
\end{tabular}

vantage of using this scale is that it can be applied repeatedly by asking for a single number while still giving reference to a number of symptoms associated with sickness. In particular this scale exploits the knowledge that nausea is generally preceded by other symptoms like dizziness, headache, (cold) sweat and stomach awareness [54], the latter symptoms varying among people in order of appearance and severity. Using this MISC, also does not require subjects to vomit as is required for rating the vomiting incidence. The MISC was rated every two minutes during all conditions. Whenever a MISC of seven or higher was scored, the condition was stopped, and the participant could return home after recovery (MISC $\leqslant 2$ ). In these cases, missing data were amended with the last rating available.

To neutralise a possible aggravation of sickness symptoms by only asking for sickness, pleasure was rated in addition. These data, however, do not contribute to the aim of this paper and are accordingly ignored.

\subsection{Mental distraction and task performance}

A double task was included to study the effect of mental distraction or cognitive workload on motion sickness also allowing for rating task performance. Because subjects were blindfolded, the input for these tasks was given aurally. To that end, each condition, lasting 20 minutes, was divided into blocks of two minutes each. During the first 110 seconds of each block subjects had to listen to a random sequence of 55 letters, one letter every two seconds. One task was to push a button each time a letter was equal to the one before. Only three letters were included: "e", "o", and "u", typically having equal phonetic characteristics and timespans (at least in Dutch), each letter thus occurring several times. Only sequences were presented with ex- actly 18 directly succeeding pairs of equal letters, each block yet using another sequence. If the button was pushed correctly within a window of $200 \mathrm{~ms}-800 \mathrm{~ms}$, the event was marked as a correct "Hit". If the button was pushed erroneously or outside the given time window, the event was marked as a "False" hit. If the button was not pushed at all, while it should have been, the event was marked as "Missed". Average reaction time over the correct Hits within each block was calculated and referred to by "RT". The second task was to count the number of hits within each block, irrespective right or wrong. During the remaining 10 seconds completing each block, subjects had to verbally report this count in addition to their MISC rating. The absolute difference between this count and the actual number was marked as "ErrMem" (= count - Hits - False). Subjects did not get any feedback on the result of their performance. Ten blocks could consequently be completed per condition. Different from the MISC, typically increasing monotonously during each motion exposure, task performance could get worse due to, e.g., sickness on the one hand, or get better due to, e.g., learning on the other. When a subject gave up prematurely due to sickness, the remaining task parameters were consequently treated as missing values.

\subsection{Subjects and procedures}

Approval by the Institutional Review Board on Experiments with Humans was obtained in accordance with the ethical standards laid down in the 1964 Declaration of Helsinki. Then, 18 paid subjects were recruited for this experiment, and their motion sickness susceptibility was rated using a motion sickness susceptibility questionnaire (MSSQ), adapted from [24]. This questionnaire asks for previous sickness occurrences in various forms of transport and fun rides for the age up to 12 , as well as for the past 12 years, resulting in a single MSSQ rating ranging from 0 (no problems whatsoever) to 222 (severe problems in all conditions). The 50th percentile of a normal population is reached at MSSQ $=37$. Candidate subjects with an MSSQ $=0$ were not considered for this experiment. All subjects included were furthermore free of obvious vestibular disorders (i.e., known by themselves), were informed about the purpose and procedures of the experiment but naive with respect to the stimulus used, signed an informed consent prior to the first experimental condition, and had not been drinking more than two alcoholic beverages during 12 hours in advance of each condition. One subject withdrew during the first 
condition due to nausea, and another withdrew just because he disliked the task. The remaining 16 subjects further reported on, accomplished all four conditions listed in Table 1 on four separate days, not more than four days in between, each day at about the same time to avoid possible diurnal effects. Eight of them were female and eight male. Ages ranged from 20 years to 61 years with an average of $31.4 \pm 14.8$. The average MSSQ was 77.2 \pm 38.6. Subjects were familiarized with the MISC and task in advance of their first condition. Apart from asking for the subjects' MISC and task ratings, there was no further communication during each condition, except that subjects were instructed to report any fast increase in sickness immediately to prevent emesis. In case no task was performed, nothing was mentioned with respect to their mental activity.

\subsection{Statistical analysis}

Statistical analysis of the current data is complicated by three factors. First, the design with respect to OVAR, head vibration, and the task performed was incomplete for reasons mentioned in Section 2.1. Secondly, despite the MISC looking like a ratio scale, it formally concerns an ordinal scale. Thirdly, task performance data might (and did) have missing values. To yet get an idea about the effects at issue, MISCdata were first separately analysed as the dependent variable by means of a parametric repeated measures ANOVA with three factors included: condition, time (or block) and subject as independent variables. This analysis hence does assume a relative degree of difference between MISC levels, and ignores possible interactions between the sub-factors OVAR, head vibration and the task performed. Subjects were included as a random factor. Post-hoc tests were performed using Tukey's criterion. The main interest regarding the task performance data, secondly, concerned their overall relationship with misery. These data were therefore analysed separately too, calculating (Pearson) correlation and significance coefficients without further reference to condition, time, and subject. When comparing means, effect sizes are calculated by their relative difference as well as the maximum likelihood estimator of Cohen's $d$.

\section{Results}

\subsection{Sickness}

Figure 3 shows the MISC data averaged over all subjects as a function of time (block) for the different con-

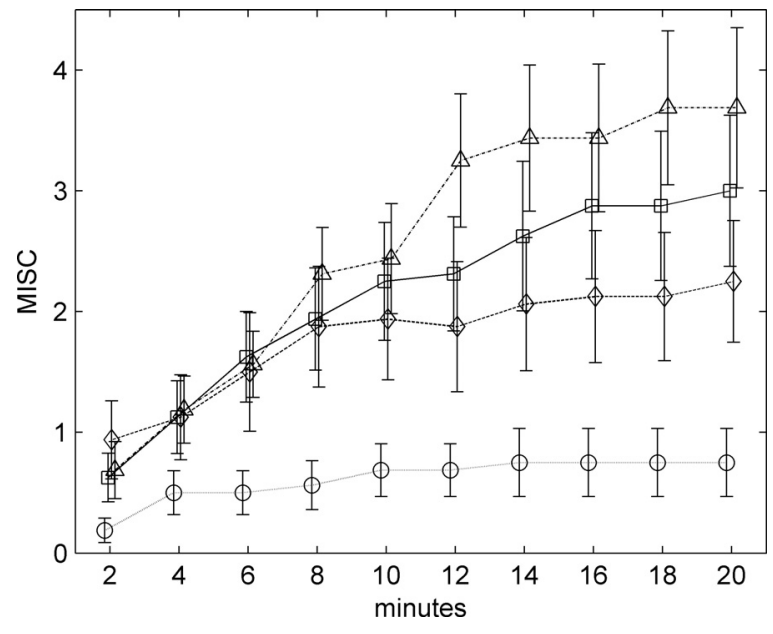

Fig. 3. MISC data averaged over all subjects per block (time interval) for the four conditions as listed in Table 1, A = Dotted line with circles (without OVAR, but with the task and head vibration), B = Solid line with squares (with OVAR and task but without the head vibration), $\mathrm{C}=$ Dashed line with diamonds (with OVAR, task, and head vibration), and $\mathrm{D}=$ Dash-dot line with triangles (with OVAR but without head vibration and task). Error bars represent standard error of mean.

ditions. Due to a minor logistic problem, the MISC was not rated at $t=0$.

(Highly) significant main effects were observed for subject $(F(15,405)=2.01, p=0.04)$, condition $(F(3,405)=5.64, p=0.002)$, block $(F(9,405)=25.9$, $p<0.0001)$. The interactions were all highly significant, i.e., subject $\times$ condition $(F(45,405)=33.4, p<$ $0.0001)$, subject $\times$ block $(F(135,405)=1.62, p=$ $0.0002)$, and condition $\times$ block $(F(27,405)=4.74$, $p<0.0001)$.

Post-hoc testing showed that all conditions differed mutually highly significantly (i.e. A vs B to D, B vs $\mathrm{C}$ to $\mathrm{D}$, and $\mathrm{C}$ vs $\mathrm{D}$ at the $1 \%$ level). The effect of time was further tested post-hoc, showing significant MISC differences (at the 1\%-level) between the first block and the third and following blocks, between the second and fourth and up, between the third and fifth and up, between the fourth and seventh and up, and the fifth and ninth and tenth blocks, thus signifying a monotonous increase. The average last recorded MISC values, i.e., at $t=20 \mathrm{~min}$, were 3.69 (OVAR only), 3.00 (OVAR + task), 2.25 (OVAR + task + vibration), and 0.75 (head vibration only). Ignoring a possible nonlinearity of the MISC scale, this implies a $25 \%$ reduction of sickness by adding a head vibration as compared to an equal condition without vibration $(d=$ 0.33 ), and a $19 \%$ reduction of sickness induced by performing a mental distracting task as compared to an 
equal condition without that task $(d=0.27)$. As compared to OVAR only, the combination of vibration and the task reduced sickness by $39 \%(d=0.61)$.

Although with each testing day a lower MISC might have been anticipated on average due to habituation, the current data did not show this effect. This (order) factor has therefore been left out in the analyses given above.

\subsection{Task performance}

None of the task performance measures showed the same clear temporal dependency as the MISC did. Moreover, about $18 \%$ of the task performance measures were missing due to premature ending of trials by sickness and a minor technical problem early on during the experiment. Table 3 shows that the MISC date correlate positively with the number of missed hits and the average reaction times, and negatively with the number of correct hits. These correlations are supported by the observation that all performance measures correlated mutually. Although the effects identified are (highly) significant, the explained variances were low.

\section{Discussion}

\subsection{Main findings}

All relevant effects shown in this study were highly significant, i.e., $p$-values typically well below 0.01 . The shortcomings of the experimental design (its incompleteness, the MISC not being a linear ratio scale, though seemingly robust against these violations, the use of OVAR, and low effect sizes observed) therefore seem less critical with respect to the following generalized conclusions. (1) Non-sickening high-frequency vibration can reduce sickness severity when added to a low-frequency sickening stimulus, (2) a mental distraction task can reduce sickness as compared to free thinking, (3) sickness can be reduced more by combining countermeasures as compared to their individual effects, and (4) task performance can be reduced by sickness. The remainder of this discussion will further elaborate on these results from a methodological, theoretical, and a practical point of view, starting off with some general remarks, and then working back towards the main vibration hypothesis elaborated in this paper.

\subsection{General remarks}

To avoid ineffective trials, candidate subjects who never experienced any sickness before were excluded.
As a result, the participants in this experiment were more susceptible to motion sickness than normal, as also indicated by the average MSSQ of 77.2 ( \pm 38.6) versus a median value of 37 for a normal population. For this reason, the effect sizes shown here, may overestimate the effects at issue, although other reasons to be discussed below may point in the opposite direction.

Contrary to other reports [12]27], no clear effect of habituation was found in the present study. This may be ascribed to the fact that the majority of MISC ratings were 5 or less, i.e., no nausea felt, although one subject withdrew from the experiment due to nausea. Although strictly speaking, "sickness" may intuitively overvalue the average symptoms observed in this study, I will still use it for reasons of simplicity, also because it is part of the motion sickness syndrome as a whole. Furthermore, in combination with the observation that the MISC ratings were not saturated on average within the 20 minute exposures used here, it might be anticipated that with a stronger stimulus and/or a longer exposure time, the effect of habituation would have become evident. Larger effect sizes may, furthermore, be anticipated when using nonblindfolded subjects. In [13], for example, the least sickness was observed in blindfolded subjects exposed to ship motion in a simulator as compared to viewing both an Earth-fixed and a subject-fixed environment. A larger effect of vibration per se may, lastly, be obtained by omitting a mental distraction task, thus allowing the subjects to "think freely". May, for this effect was only tested in conditions with a task (see further below).

Chair and headrest rotation in this experiment were perpendicular, thus allowing additional sickening cross-coupled or Coriolis stimulation. Although the effect thereof has likely been negligible due to the small vibration amplitude and its high frequency, the positive effect of head vibration as such would have even been larger in the absence of the Coriolis effect, e.g., when induced by mere linear acceleration.

The question whether the effect of vibration would be bigger in case of stronger and/or longer sickness provoking stimuli, lastly, may be countered by the possibility that the effect of vibration is limited to the absolute maximum obtained in this experiment already. With more severe sickness, the overall relative effect of vibration would then be less. That, however, remains to be seen, while possibilities to increase the effect of vibration are discussed in the last section. Moreover, it can also be assumed that even a small initial effect may prevent further accumulation of symptoms, thus resulting in a relatively large net effect at the end. 
Table 3

Correlation coefficients $(r)$ and significance levels $(p, p<0.001$ rounded down to zero) of the relations between the MISC and the task performance measures and the task performance measures mutually

\begin{tabular}{|c|c|c|c|c|c|c|c|c|c|c|}
\hline & \multicolumn{2}{|c|}{ Hits } & \multicolumn{2}{|c|}{ Missed } & \multicolumn{2}{|c|}{ False } & \multicolumn{2}{|c|}{ RT } & \multicolumn{2}{|c|}{ ErrMem } \\
\hline & $r$ & $p$ & $r$ & $p$ & $r$ & $p$ & $r$ & $p$ & $r$ & $p$ \\
\hline MISC & -0.15 & 0.003 & +0.15 & 0.003 & n.s. & & +0.11 & 0.03 & n.s. & \\
\hline Hits & & & -0.67 & 0 & -0.79 & 0 & -0.53 & 0 & n.s. & \\
\hline Missed & & & & & +0.1 & 0.03 & +0.2 & 0 & +0.18 & 0 \\
\hline False & & & & & & & +0.54 & 0 & -0.17 & 0.01 \\
\hline RT & & & & & & & & & -0.17 & 0 \\
\hline
\end{tabular}

\subsection{Task performance}

Over all conditions, task performance decreased with observed misery ratings. The number of correctly indicated appearances of two equal successive letters decreased with increasing sickness, while the number of missed combinations as well as the reaction time required for the indications increased with increasing misery. The task itself seemed to be appropriate in this respect, the different indices all showing a (mutually) consistent behaviour as shown by the data in Table 3 .

Although the effects of misery on task performance were significant, the sizes thereof were limited. Larger effects may, however, be anticipated in cases of more severe sickness than observed in the present experiment. Moreover, mental distraction can then be anticipated to have a larger positive effect on task performance as well. Performing a task in sickening conditions therefore seems to be an inherently effective way of improving the performance thereof in sickening conditions: the task leading to less sickness, which reduction in turn improves the performance of the task itself (although strictly speaking no improvement is possible is no task is performed).

\subsection{Distraction}

Distraction from contemplating on sickness did reduce the amount of sickness observed in this experiment significantly. This is in accordance with data presented by [29], but seems to oppose those found by [60], the latter comparing autogenic feedback training with a "neutral" cognitive task. They, however, applied a between subjects comparison, a smaller sample size, and no information was given on the no-task circumstances as opposed to playing blackjack as the distracting task. Their data therefore not necessarily need to contradict the current observations. The effect of listening to (pleasant) music [39]63], may furthermore be considered a matter of mental distraction as well. Given the few experimental data available on this topic, the current overall score is in favour of concluding that distraction from contemplating on sickness per se is beneficial with respect to motion sickness.

The most practical consequence of performing a task during sickening conditions has already been mentioned above, i.e., performing a task in sickening conditions will lead to less sickness, which in turn improves the performance of the task itself (although strictly speaking no improvement is possible if no task is performed). Paying too much parental attention to children susceptible to car sickness, for example, therefore seems unwise. Even the act of supplying antimotion sickness drugs complemented with a phrase like "you will probably get sick, so take your pill", could already dispose the victim to sickness beforehand, possibly cancelling the positive effect of that drug. Although [39] did not find an effect of prior expectations on subsequent motion sickness severity, this idea yet seems to be supported by [65]. In military environments, furthermore, the social culture probably already prevents those susceptible from focussing too much on their sickness, which, in this particular case thus seems to be a beneficial attitude. This, however, does not imply that sickness should be ignored completely, for it is an issue regarding performance in general [4 8448], and treatment can be helpful in that respect, which is why this paper also further studied a novel possibility to do so.

\subsection{Vibration}

As stated in the introduction, functioning organs of balance are essential in the genesis of motion sickness, because people without, generally do not get sick from motion [35]48]. The vibration was therefore applied directly to the head in this experiment, and not to the seat stimulating mainly the torso, for example. The observation that a vibration applied to the head did affect sickness thus supports the idea that the organs of balance do play a role in the genesis of motion sickness. It likewise questions the role of abdominal tissue in the genesis of motion sickness, as suggested by [24]. Fur- 
thermore it nuances the assumption described in [55], that postural instability is a necessary and sufficient condition for getting sick from motion (see also [5]). The subjects in the present experiment were not able to counter the OVAR motion in any way, which is why postural stability cannot have been the leading factor.

It was assumed in the introduction that high-frequency vibration per se is not sickening (i.e., above $1 \mathrm{~Hz}$,

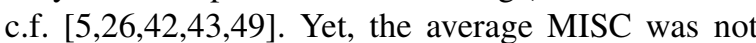
zero in the control condition with the head vibration only. However, this average did level off after some 10 minutes about a value of less than 1 , which only implies some discomfort according the definition given in Table 2, not necessarily being related to sickness symptoms. It therefore seems reasonable to assume that the vibration as used in this experiment, although slightly uncomfortable, is not sickening as such.

In a literal sense, "less sickness with more motion" has also been observed in [19], where they observed more head and torso motion and less sickness in subjects actively driving a virtual vehicle than in subjects watching equal motion passively. In that study, however, no distinction was made regarding motion frequency, as is the crucial point in the current paper. The motions experienced by the drivers in their experiment could thus have been more sickening per se than those experienced by their passengers. Moreover, a fourfold(!) difference was observed in [22] between passengers without an external view and those faced with a trajectory to be followed, showing that anticipation seems to be an essential factor explaining the difference observed by [19]. This explanation also nicely fits the observer theory further elaborated in the next section.

Although the parsimonious explanation that vibration may have served as a mere mental distractor cannot be ruled out, it is just like the explanation that it serves as an attractor. Moreover, a basic rational explanation is at hand, as discussed below.

\subsection{Theoretical implications}

Although rather hypothetical, it may be predicted that there is a limit to the reduction of low-frequency induced sickness by the addition of a high-frequency vibration. The assumption outlined in the introduction states that motion sickness may be caused by a conflict between a sensed and a predicted estimate of verticality, and that this conflict is dominated by a phase lag due to a process making a distinction between gravitational and inertial accelerations [3,5,-7,9]. The conflict in this theory, and hence the amount of sickness, can be attenuated by decreasing an internal feedback or Kalman [58] gain. A reduced gain, as stated in the introduction, may in turn be caused by increasing the noise and/or uncertainty of the sensory signals by, e.g., adding a high-frequency non-sickening vibration. Even in the ultimate condition when the feedback gain, and hence the orientation of the predicted estimate of verticality would be invariably upright, the conflict would still be non-zero due to persistent variation of sensory signals. Here, a maximum conflict may be assumed in case of a $180^{\circ}$ phase difference between the sensed and expected verticals, and a zero conflict when the sensed and expected signals are equal. The maximum theoretical reduction in the sickness causing conflict due to the elimination of the internal feedback gain would then be $50 \%$, at least in a first order approximation ignoring nonlinearities in the conflict to sickness transformation and misery ratings. This assumption seems to be substantiated by the observations by [33] and even more so by [22]. The latter presented congruent visual information to subjects experiencing low-frequency sickening physical motion in a visually enclosed cabin, and were able to reduce sickness by over a factor of four as compared to the condition without visual information. Assuming that within the central nervous system, visual and vestibular information converge only after the low-pass filtering making the distinction between gravity and inertia, and assuming a non-zero Kalman gain in this case, the difference between the expected and sensed verticals may then theoretically approach zero. Although likely not attainable in practice, a reduction of over a factor of two seems possible in this case, as indeed was shown. Although not proven, this reasoning does constitute a consistent framework explaining the results observed, including those presented in this paper.

An interesting thought concerns the validation of the assumption that the time constant of the so called velocity storage mechanism is reduced by adding vibration too. Here, velocity storage refers to the neural mechanism extending the time constant of the decay of angular motion perception and eye movements after a change in angular motion [52]. It has been shown that this time constant is longer in aviators susceptible to motion sickness [8]. Moreover, [47] have shown that velocity storage can be explained by an equal observer model as discussed above, and that the time constant thereof increases with an increase of the internal feedback gain. Combining these observations, it may be concluded that a reduction of the feedback gain 
induced by, e.g., vibration, would indeed result in a shorter time constant of the velocity storage mechanism, and hence in less sickness as observed. To further validate this hypothesis, the same vibration as described here might therefore be applied in an experiment rotating subjects about an Earth vertical axis with a fast acceleration and deceleration, measuring the time constant of the vestibulo-ocular reflex (the main parameter as studied by [47]), and/or the perception of angular self-motion. If a reduction of the time constant by added vibration then is observed, this would favour the observer theoretical explanation of the phenomena at issue here.

If, furthermore, the observer model explanation of motion sickness and the reduction thereof by vibration were right, this could imply an increase of the positive main effect found here at longer exposure times. By "learning" that the input signal is degraded by noise, the Kalman gain may be further optimised continually, in this case decreased, to decrease the error signal leading to sickness. Another example of the possible variability of the Kalman gain may be given by the (personal) observation that within the same individual small motions not sickening aboard a ship where motion is anticipated with the consequence of a low gain, may be sickening in a tall building where no motion is anticipated with the consequence of a high gain. Although this hypothesis awaits further validation, again it adds to the attractiveness of the framework as a whole to explain the phenomena at issue.

A last comment on the observer model referred to above concerns its possible use of cognitive inputs, explaining the effect of distraction in addition. In the experiment described in this paper, the task related cognitive inputs were not related to the motion at issue in any way, thus being unable to optimise the predicted sense of verticality. The effect of mental distraction therefore seems to be less likely caused by an altered feedback and ensuing conflict, but more likely by, e.g., an increased threshold in the vegetative (gastro-intestinal) symptoms driven by that conflict. Such a post-conflict pathway has been suggested before, explaining for example the accumulation and gradual decrease of symptoms during and after a pulse wise modulated stimulus [6]750:51].

\subsection{Application}

Irrespective of these explanations, the observation that the addition of a high-frequency vibration to a lowfrequency sickening stimulus reduces the amount of sickness as such, may yet have several practical consequences. One consequence is that this observation nuances the general pursuit for reducing vehicle vibration to the technical possible minimum, while from a perceptual point of view some vibration may be beneficial. This may be exemplified by the said sickness provoking nature of some cars, especially those with a reputation on smooth (air) suspension. In ships, lastly, it has been shown that seasickness is the number one cause of discomfort [11], and engine vibration might thus increase the comfort. Yet, not all people do always suffer from sickness, which is why in most cases, comfort in general will probably still be higher with less vibration.

Returning to my personal experience, the most obvious consequence probably concerns the simple advise to lean the head against a (not too vigorously) vibrating car or bus window when feeling sick. A vibrating device attached to the head, possibly provided with a frequency and amplitude adjustable to the user's own preference, may give an alternative for medication, also in conditions lacking such a vibrating vehiclefixed structure. In that case, the question also becomes relevant whether an optimisation of the effect is possible by using different vibration characteristics, both with respect to frequency and amplitude, and the way of application. In this experiment, for example, the force by which the subjects pushed their heads into the head rest was not controlled, and the true variability of RMS accelerations may have been different from those reported. This variability may thus also explain part of the variability in the reduction of misery in this experiment. A more tight connection between a vibrating unit and the skull (or teeth, although physically optimal, probably not preferable with respect to comfort), may therefore also be considered. In addition it may be considered to only apply the vibration intermittently and/or on demand. The latter may also be desirable when performing a visual task possibly affected by a reduced visual acuity induced by the vibration. In all cases, health risks should, of course, be taken into account. Although explicit limit values for head vibration are not given by [34], the limits mentioned in the Methods section may serve this purpose.

A very last remark concerns the statement in the Introduction that there does not seem to be one single countermeasure eliminating all symptoms of motion sickness, but a combination of remedies may be satisfactory. Here it is shown that vibration and mental distraction reduce sickness by 25 and $19 \%$, respectively as such, and reduce sickness by $39 \%$ when com- 
bined, thus showing that the alleviating effects of multiple remedies against motion sickness can be mutually strengthening. The here described novel method of applying vibration therefore adds to the battery of existing remedies that, although possibly causing a marginal effect by themselves, may give a significant reduction of sickness and improvement of performance when combined.

\section{Acknowledgments}

This work was facilitated by the Dutch Ministry of Defence, within the framework of the project V937 Improved Performance at Motion. The author, however, does not have a financial relationship with this organisation, nor does he have a conflict of interests. TNO colleagues Martin van Schaik, Natasja van der Leden and Simone Brouwer conducted the experiment, Frank Geurtsen programmed the letter task and Mark Houben gave critical and keen comments on the draft of this paper.

\section{References}

[1] A.J. Benson, Motion sickness, in: Aviation Medicine, 2nd ed., J. Ernesting and P. King, eds, Butterworths and Co, Boston, 1988, pp. 318-338

[2] L.E. Bertolucci and B. DiDario, Efficacy of a portable acustimulation device in controlling seasickness, Aviation, Space, and Environmental Medicine 66 (1995), 1155-1158.

[3] W. Bles, J.E. Bos, B. de Graaf, E. Groen and A.H. Wertheim, Motion sickness: Only one provocative conflict? Brain Research Bulletin 47 (1998), 481-487.

[4] J.E. Bos, How motions make people sick such that they perform less: A model based approach, NATO RTO/AVT-110 Symposium on Habitability of Combat and Transport Vehicles: Noise, Vibration and Motion, Prague, CZ, 4-7 October 2004, 27/1-11.

[5] J.E. Bos, Nuancing the relationship between motion sickness and postural stability, Displays 32 (2011), 189-193.

[6] J.E. Bos and W. Bles, Modelling motion sickness and subjective vertical mismatch detailed for vertical motions, Brain Research Bulleting 47 (1998), 537-542.

[7] J.E. Bos and W. Bles, Theoretical considerations on canalotolith interaction and an observer model, Biological Cybernetics 86 (2002), 191-207.

[8] J.E. Bos, W. Bles and B. de Graaf, Eye movements to yaw, pitch, and roll about vertical and horizontal axes: Adaptation and motion sickness, Aviation, Space, and Environmental Medicine 73 (2002), 436-444.

[9] J.E. Bos, W. Bles and E.L. Groen, A theory on visually induced motion sickness, Displays 29 (2008), 47-57.

[10] J.E. Bos, J.L. Colwell and A.H. Wertheim, A focus on motion sickness regarding the 1997 NATO performance assessment questionnaire (PAQ) data, TNO Report, TNO Human Factors, Soesterberg, the Netherlands, 2002, TM-02-A017.
[11] J.E. Bos, D. Damala, C. Lewis, A. Ganguly and O. Turan, Susceptibility to seasickness, Ergonomics 50 (2007), 890-901.

[12] J.E. Bos, S.C. de Vries, M.L. van Emmerik and E.L. Groen, The effect of internal and external fields of view on visually induced motion sickness, Applied Ergonomics 41 (2010), 516-521.

[13] J.E. Bos, S.N. MacKinnon and A. Patterson, Motion sickness symptoms in a ship motion simulator: Effects of inside, outside, and no view, Aviation, Space, and Environmental Medicine 76 (2005), 1111-1118.

[14] D.G. Bruce, J.F. Golding, N. Hockenhull and R.J. Pethybridge, Acupressure and motion sickness, Aviation, Space, and Environmental Medicine 61 (1990), 361-365.

[15] B.S.K. Cheung, I.P. Howard and K.E. Money, Visuallyinduced sickness in normal and bilaterally labyrinthinedefective subjects, Aviation, Space, and Environmental Medicine 62 (1991), 527-531.

[16] M.J. Correia and F.E. Guedry, Modification of vestibular responses as a function of rate of rotation about an earth horizontal axis, Acta Otolaryngologica 62 (1966), 297-308.

[17] P. Denise, C. Darlot, J. Droulez, B. Cohen and A. Berthoz, Motion perceptions induced by off-vertical axis rotation (OVAR) at small angles of tilt, Experimental Brain Research 73 (1988), 106-114.

[18] T.G. Dobie and J.G. May, Cognitive-behavioral management of motion sickness, Aviation, Space, and Environmental Medicine Supplement 65 (1994), C1-C20.

[19] X. Dong, K. Yoshida and T.A. Stoffregen, Control of a virtual vehicle influences postural activity and motion sickness, Journal of Experimental Psychology: Applied 17 (2011), 128-138.

[20] M.L. van Emmerik, S.C. de Vries and J.E. Bos, Internal and external fields of view affect cybersickness, Displays 32 (2011), 169-174.

[21] European Union, Directive 2002/44/EC of the European Parliament and of the Council of 25 June 2002 on the minimum health and safety requirements regarding the exposure of workers to the risks arising from physical agents (vibration), sixteenth individual Directive within the meaning of Article 16(1) of Directive 89/391/EEC, 2002.

[22] P.J. Feenstra, J.E. Bos and R.N.H.W. van Gent, A visual display enhancing comfort by counteracting airsickness, Displays 32 (2011), 194-200.

[23] J. Förstberg, E. Andersson and T. Ledin, Influence of different conditions for tilt compensation on symptoms of motion sickness in tilting trains, Brain Research Bulletin 47 (1998), 525-535.

[24] H.E. von Gierke and D.E. Parker, Differences in otolith and abdominal viscera graviceptor dynamics: Implications for motion sickness and perceived body position, Aviation, Space, and Environmental Medicine 65 (1994), 747-751.

[25] J.F. Golding, Motion sickness susceptibility questionnaire revised and its relationship to other forms of sickness, Brain Research Bulletin 47 (1998), 507-516.

[26] J.F. Golding, D. Phil, A.G. Mueller and M.A. Gresty, A motion sickness maximum around the $0.2 \mathrm{hz}$ frequency range of horizontal translational oscillation, Aviation, Space, and Environmental Medicine 72 (2001), 188-192.

[27] J.F. Golding and J.R.R. Stott, Objective and subjective time courses of recovery from motion sickness assessed by repeated motion challenges, Journal of Vestibular Research 7 (1997), 421-428.

[28] A. Grontved, T. Brask and J. Kambskard, Ginger root against seasickness: A controlled trial on the open sea, Acta Otolaryngologica 105 (1988), 45-49. 
[29] F.E. Guedry, Visual control of habituation to complex vestibular stimulation in man, Acta Otolaryngologica 58 (1964), 377-389.

[30] F.E. Guedry and E.K. Montague, Quantitative evaluation of the vestibular coriolis reaction, Aerospace Medicine 32 (1961), 487-500.

[31] J. Hill, The care of the sea-sick, British Medical Journal OctDec (1936), 802-807.

[32] J.E. Holly, S.J. Wood and G. McCollum, Phase-linking and the perceived motion during off-vertical axis rotation, Biological Cybernetics 102 (2010), 9-29.

[33] M.M.J. Houben and J.E. Bos, Reduced seasickness by an artificial 3d earth-fixed visual reference, Proceedings International Symposium on Human Performace At Sea, Glasgow, (16-19 June 2010), 263-270.

[34] International organization for standardization, mechanical vibration and shock-evaluation of human exposure to wholebody vibration-Part 1: General requirements, ISO, 2631$1: 1997(\mathrm{E})$.

[35] J.A. Irwin, The pathology of seasickness, The Lancet 2 (1881), 907-909.

[36] W.H. Johnson, F.A. Sunahara and J.P. Landolt, Importance of the vestibular system in visually induced nausea and selfvection, Journal of Vestibular Research 9 (1999), 83-87.

[37] D.R. Jones, R.A. Levy, L. Gardner, R.W. Marsh and J.C. Patterson, Self-control of psychophysiologic response to motion stress: Using biofeedback to treat airsickness, Aviation, Space, and Environmental Medicine 56 (1985), 1152-1157.

[38] E.E. Jozsvai and R.A. Pigeau, The effect of autogenic training and biofeedback on motion sickness tolerance, Aviation, Space, and Environmental Medicine 67 (1996), 963-968.

[39] R.S. Kennedy, D.S. Lanham, J.M. Drexler, C.J. Massey and M.G. Lilienthal, A comparison of cybersickness incidences, symptom profiles, measurement techniques, and suggestions for further research, Presence: Teleoperators and Virtual Environments 6 (1997), 638-644.

[40] B. Keshavarz and H. Hecht, Validating an efficient method to quantify motion sickness, Human Factors 53 (2011), 415426.

[41] B. Keshavarz and H. Hecht, Pleasant music as a countermeasure against visually induced motion sickness, Applied Ergonomics 45 (2013), 521-527.

[42] J.R. Lackner and P. DiZio, Space motion sickness, Experimental Brain Research 175 (2006), 377-399.

[43] A. Lawther and M.J. Griffin, The motion of a ship at sea and the consequent motion sickness amongst passengers, $E r$ gonomics 29 (1986), 535-552.

[44] A. Lawther and M.J. Griffin, A survey of the occurrence of motion sickness amongst passengers at sea, Aviation, Space, and Environmental Medicine 59 (1988), 399-406.

[45] H.C. Lien, W.M. Sun, Y.H. Chen, H. Kim, W. Hasler and C. Owyang, Effects of ginger on motion sickness and gastric slow-wave dysrhythmias induced by circular vection, American Journal of Physiology - Gastrointestinal Liver Physiology 284 (2003), G481-G489.

[46] J.B. Lucot, Pharmacology of motion sickness, Journal of Vestibular Research 8 (1998), 61-66.

[47] J.A. McClure and A.R. Fregly, Effect of environmental temperature on sweat onset during motion sickness, Aerospace Medicine 43 (1972), 959-967.

[48] D.M. Merfeld, L.R. Young, C.M. Oman and M.J. Shelhamer, A multidimensional model of the effect of gravity on the spa- tial orientation of the monkey, Journal of Vestibular Research 3 (1993), 141-161

[49] K.E. Money, Motion sickness, Physiological Reviews 50 (1970), 1-39

[50] J.F. O'Hanlon and M.E. McCauley, Motion sickness incidence as a function of the frequency and acceleration of vertical sinusoidal motion, Aerospace Medicine 45 (1974), 366369.

[51] C.M. Oman, A heuristic mathematical model for the dynamics of sensory conflict and motion sickness, Acta Otolaryngological Supplement 392 (1982), 1-44.

52] C.M. Oman, Motion sickness: A synthesis and evaluation of the sensory conflict theory, Canadian Journal of Physiology and Pharmacology 68 (1990), 294-303.

[53] T. Raphan, V. Matsuo and B. Cohen, Velocity storage in the vestibulo-ocular reflex arc (VOR), Experimental Brain Research 35 (1979), 229-248.

[54] J.T. Reason and J.J. Brand, Motion sickness, Academic Press, London, 1975.

[55] M.F. Reschke, J.T. Somers and G. Ford, Stroboscopic vision as a treatment for motion sickness: Strobe lighting vs. shutter glasses, Aviation, Space, and Environmental Medicine 77 (2006), 2-7.

[56] G.E. Riccio and T.A. Stoffregen, An ecological theory of motion sickness and postural instability, Ecological Psychology 3 (1991), 195-240.

[57] A. Rolnick and W. Bles, Performance and well-being under tilting conditions - The effects of visual reference and artificial horizon, Aviation, Space, and Environmental Medicine 60 (1989), 779-785.

[58] R.S. Schwab, The nonlabyrinthine causes of motion sickness, International Record of Medicine 167 (1954), 631-637.

[59] P. Selva and C.M. Oman, Relationships between observer and Kalman filter models for human dynamic spatial orientation, Journal of Vestibular Research 22 (2012), 69-80.

[60] R. Simons and J. Bos, Medicine against motion sickness: Effectiveness and safety, Nederlands Militair Geneeskundig Tijdschrift 61 (2008), 77-90.

[61] W.B. Toscano and P.S. Cowings, Reducing motion sickness: A comparison of autogenic-feedback training and an alternative cognitive task, Aviation, Space, and Environmental Medicine 53 (1982), 449-453.

[62] M. Turner and M.J. Griffin, Motion sickness in public road transport: Passenger behaviour and susceptibility, Ergonomics 42 (1999), 444-461.

[63] M. Turner, M.J. Griffin and I. Holland, Airsickness and aircraft motion during short-haul flights, Aviation, Space, and Environmental Medicine 71 (2000), 1181-1189.

[64] F.D. Yen Pik Sang, J.P. Billar, J.F. Golding and M.A. Gresty, Behavioural methods of alleviating motion sickness: Effectiveness of controlled breathing and a music audiotape, Journal of Travel Medicine 10 (2003), 108-111.

65] F.D. Yen Pik Sang, J.F. Golding and M.A. Gresty, Suppression of sickness by controlled breathing during mildly nauseogenic motion, Aviation Space and Environmental Medicine 74 (2003), 998-1002.

[66] S.D. Young, B.D. Adelstein and S.R. Ellis, Demand characteristics in assessing motion sickness in a virtual environment: Or does taking a motion sickness questionnaire make you sick? IEEE Transactions on Visualization and Computer Graphics 13 (2007), 422-428. 